\title{
Towards Match-on-Card Finger Vein Recognition
}

\author{
Michael Linortner \\ Andreas Uhl \\ \{mlinortner,uhl\}@cs.sbg.ac.at \\ University of Salzburg, Department of Computer Sciences \\ Salzburg, Austria
}

\begin{abstract}
Security and privacy is of great interest in biometric systems which can be offered by Match-on-Card (MoC) technology, successfully applied in several areas of biometrics. In finger vein recognition such a system is not available yet. Utilizing minutiae points from vein images in combination with classical minutiae-based fingerprint comparison software offers a great opportunity to integrate vein recognition on $\mathrm{MoC}$ systems. In this work a publicly available and two commercial fingerprint comparison tools are used to evaluate the recognition performance of vein minutiae, represented in a standardized data format, on three publicly available databases. The results strongly indicate that minutiae-based comparison technology from fingerprint recognition can be applied to finger vein recognition and is able to compete with and even outperform classical correlation-based methods utilized in this field. The work done here prepares the way for vein recognition on MoC systems.
\end{abstract}

\section{CCS CONCEPTS}

- Security and privacy $\rightarrow$ Biometrics.

\section{KEYWORDS}

Biometrics; Finger vein recognition; minutiae; match on card; MoC; minutiae-based comparison; minutiae-based vein recognition

ACM Reference Format:

Michael Linortner and Andreas Uhl. 2021. Towards Match-on-Card Finger Vein Recognition. In Proceedings of the 2021 ACM Workshop on Information Hiding and Multimedia Security (IH\&MMSec '21), June 22-25, 2021, Virtual Event, Belgium. ACM, New York, NY, USA, 6 pages. https://doi.org/10.1145/ 3437880.3460406

\section{INTRODUCTION}

Finger vein recognition [18] has become a mature biometric trait and alternative to fingerprint recognition. Classical techniques in finger vein recognition, like principal curvature (PC) [7] or the usage of Gabor filters [10], use segmentation methods to extract a binary representation of the veins. For template comparison a similarity score between two binary feature templates is calculated using correlation. Another approach is to use local information like minutiae points of the veins analogous to minutia points used in fingerprint recognition.

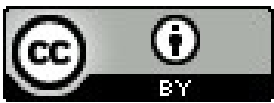

This work is licensed under a Creative Commons Attribution International 4.0 License. IH\&MMSec '21, June 22-25, 2021, Virtual Event, Belgium.

(C) 2021 Copyright held by the owner/author(s).

ACM ISBN 978-1-4503-8295-3/21/06.

https://doi.org/10.1145/3437880.3460406
Contribution of this work: Security and privacy is of great interest in using biometric systems. MoC technology offers significant advantage in convenience, security and performance and is becoming an integral part of high-security smart cards [4]. As far as the authors are aware no MoC system exists for finger vein recognition yet. For fingerprint recognition on the other hand, there is a considerable amount of minutiae-based $\mathrm{MoC}$ solutions $[6,16]$ available. Provided the minutiae-based approach investigated in this work yields high accuracy, a seamless integration of finger vein minutiae into MoC systems is possible, which further means this is the first MoC system in vein recognition. Therefore, in this work minutiae-based fingerprint template comparison is employed on minutiae points obtained from finger vein images using commercial software, whose vendors also offer MoC solutions (see section 3). The minutiae are stored in a standardized biometric data format.

Related Work: Previous work related to usage of finger vein minutiae includes [23] where comparison of two minutiae point sets is done by measuring Modified Hausdorff distance (MHD). In [14] minutiae points are extracted from several images and combined to one point set. To select the 'most reliable' points a pruning method based on Genetic algorithm is introduced. For comparison MHD with $\mathrm{k}$ nearest neighbors is adopted. Local binary descriptors to describe minutiae points are used in [11]. A singular value decomposition (SVD) based minutiae pairing is executed and based on the descriptors false pairs are removed and comparison scores are computed. In [21] the binary vein structure in a local environment around detected minutiae points is used to filter out possible impostors during the comparison stage. For the final score a correlationbased template comparison is performed on the whole vein image. A location-based spectral minutiae representation (SML) is calculated from vein minutiae points in [15]. The obtained spectra are compared using a correlation-based measure. Originally SML has been proposed for fingerprint minutiae recognition [20]. In our previous work [5] the usage of commercial fingerprint comparison software on finger vein minutiae has been investigated, but the resulting equal error rate (EER) is rather poor and is considerably improved in this work using a novel approach to extract vein minutiae. This work follows the same evaluation protocol but in contrast to the previous work here a different image enhancement, vein segmentation and minutiae extraction technique is employed. This approach leads to a considerable improved recognition performance.

\section{FEATURE EXTRACTION}

Analogous to bifurcations in a fingerprint, intersections and branches in the vein structure can be retrieved as minutiae points. To extract them from the vein image several processing steps are carried out: 


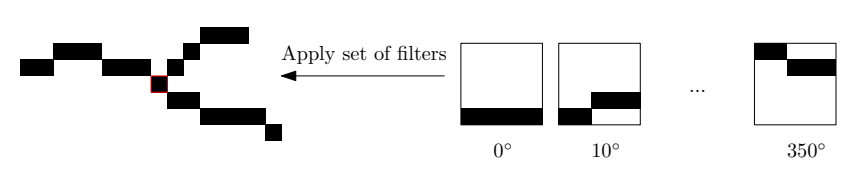

Figure 1: A set of 'direction filters' applied to a skeleton branch at a bifurcation point.

first region of interest (ROI) extraction, then preprocessing and image enhancement, followed by vein segmentation, thinning, and minutiae extraction. A fixed sized ROI is cut out from the original images using an approach based on [12]. The acquired vein images are blurry and tend to have a low contrast. In this work speeded-up adaptive contrast enhancement (SUACE) [3] is used to deal with the varying contrast present in the images. After enhancement, the images are downscaled by a factor of two.

For vein segmentation PC [7] is used, which tends to segment the veins as thick solid lines which eases the process of thinning. After PC the morphological operations opening and closing are applied to remove small islands and to close small holes which could be considered as noise. Subsequently thinning is applied to retrieve a skeleton of the vein pattern using a method from Zhang and Suen [24].

For detecting the minutiae points each pixel $p$ of the skeleton is investigated and its crossing number $c n(p)$ is calculated [2]:

$$
c n(p)=\frac{1}{2} \sum_{i=1}^{8}\left|n_{k}-n_{k+1}\right|
$$

where $n_{k} \in\{0,1\}$ and $n_{9}=n_{1}$ are the pixels of the 8-neighborhood of $p$. This number indicates whether a pixel is an end, bifurcation, or crossing point with $c n(p)=1, c n(p)=3$, and $c n(p)>3$ respectively [13].

The direction $\theta$ of a bifurcation point is retrieved following the ANSI/INCITS 378-2004 standard [1]: the direction points along the bisection of the smallest of the three angles enclosed by the legs of the bifurcation skeleton. To extract $\theta$, a set of 36 'direction filters' with window size $k=7$ pixels is applied, each filter representing a line with an orientation between 0 and 350 degrees, see figure 1. The three highest responses indicate the direction of the legs of a bifurcation. For crossings no direction is estimated. No quality assessment is done for the minutiae points. Figure 2 illustrates the whole minutiae extraction process.

The extracted minutiae are stored in the ANSI/INCITS 378-2004 [1] data format, which is required for the usage with commercial minutiae-based comparison solutions. The data format demands a value for the resolution of the image from which the minutiae points have been extracted. Some tests showed that with varying values comparison modules may produce different output. Considering the usage of fingerprint recognition tools, the value should somehow reflect an equivalent relation between resolution and spread of the minutiae points across the image as in typical fingerprint images. The following consideration has been made: The bounding box of a fingerprint with an optimal $500 \mathrm{dpi}$ resolution should be of the same size as the ROI of the vein images. After analyzing fingerprints taken with several sensors it has been determined that the longer side of the bounding box measures $2.5 \mathrm{~cm}$ and the resolution value

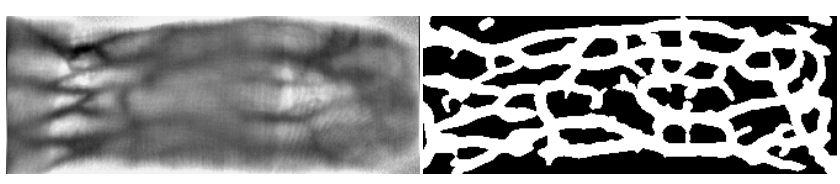

(a) SUACE

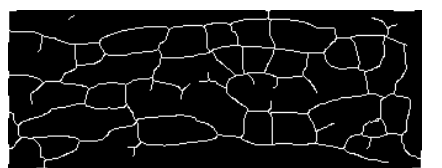

(c) Thinned (b) PC (d) Minutiae

Figure 2: Example image from the UTFVP data set visualizing the process of minutiae extraction: (a) shows the enhanced image, (b) the segmented veins after applying PC, (c) the vein skeleton and (d) the extracted minutiae points with their assigned direction.

can be derived. Standardized minutiae data records are created using the BiomDI ${ }^{1}$ software tool.

\section{EXPERIMENTS}

The experiments' aim is to show that the accuracy achieved with finger vein minutiae points evaluated on fingerprint comparison software in contrast to classical vein recognition techniques or $\mathrm{CNN}$ based approaches is high enough to enable a practicable usage of vein minutiae on a MoC system.

Databases: Three publicly available finger vein databases were used for the experiments:

(1) University of Twente Finger Vascular Pattern Database (UTFVP) [17] consists of 4 sample images for each of the 6 fingers from 60 subjects.

(2) PLUSVein-FV3 Finger Vein Database [8] contains four data sets with images from 60 subjects and 6 fingers per subject captured from the dorsal and palamr view, both acquired under LED and Laser illumination. Each data set contains 5 samples of each finger. In this work only the results for the data sets acquired under LED illumination are reported because the results for both illuminations turned out to be almost similar. The data sets are denoted as PLUSLed-P and PLUS-Led-D for palmar and dorsal view, respectively. The provided ROIs are used in the experiments.

(3) HKPU-FV [10] contains images of 156 subjects with 2 finger per user and 6 samples per finger. In our experiments only session one is considered, because there are the most users present.

Minutiae-based methods: For minutiae-based comparison the publicly available Bozorth3 as part of the NIST Biometric Image Software (NBIS) Release 5.0.0 2 and two state of the art commercial products, the IDKit SDK Version $9.0^{3}$ and the VeriFinger 11.2 Extended $\mathrm{SDK}^{4}$ have been used. The vendors of latter both offer

\footnotetext{
${ }^{1}$ https://www.nist.gov/services-resources/software/

biomdi-software-tools-supporting-standard-biometric-data-interchange

${ }^{2}$ https://www.nist.gov/services-resources/software/nist-biometric-image-softwarenbis

${ }^{3}$ https://www.innovatrics.com

${ }^{4}$ https://www.neurotechnology.com/verifinger.html
} 
a MoC system ${ }^{5}$. Additionally MHD and SML, which were used in $[14,15,23]$, are evaluated. For template comparison in SML a cross-correlation of the z-score normalized spectra has been used.

Established vein recognition comparison methods: In contrast to the minutiae-based methods three classical approaches were employed directly on the preprocessed vein images. The first method was PC [7] which uses a correlation based comparison. As second method a Gabor filter based approach (GF), presented in [10], has been used. Due to the lack of a publicly available reference implementation and some inconsistency in the description of the comparison step, we adapted it and a dissimilarity score between two binary templates $R$ and $T$ is calculated as follows: The pixels which are different within a ROI in $R$ and $T$ are counted and divided by the size of the ROI. The ROI is the intersection of the mask $M_{R}$ and $M_{T}$, which denote the finger region in $R$ and $T$, respectively. ASAVE [22] was used as third method, which uses a curvature-based vein segmentation approach to generate two templates, which are used in the comparison stage to compute a combined score based on correlation. On all methods a horizontal and vertical shift as well rotation of the template was applied to compensate for misalignment.

CNN based vein recognition: In [19] CNN based methods are applied on the same data sets used in this work. The authors of [19] used an extended version of the PLUS data set and only the palmar view. They used PC as one of their comparison methods and its performance is close to the results of PC in our work. Therefore, we take the result values of the best performing CNN method from their work and include it in this paper as additional comparison method.

Parameter search and Evaluation: The quality of the segmented veins heavily depends on image quality and on the parameters chosen for preprocessing or the vein segmentation algorithms. Each sensor produces images in different quality. Therefore, for each available data set the methods and parameters are selected individually. The correlation-based comparison techniques are also influenced by the chosen parameters. So far in literature there is no generally accepted process to derive these parameters.

Each finger of an individual is treated as a single class. To compute comparison scores the fingerprint verification competition $2006^{7}$ (FVC) protocol has been employed to reduce the amount of impostor comparisons: Each sample of a finger is compared to each remaining sample of the same finger for genuine comparisons, whereas for impostor comparisons only the first sample of each finger is compared to each first sample of the remaining fingers. Symmetric comparisons are not considered, e.g. if sample $\mathrm{x}$ is compared to sample $\mathrm{y}$, $\mathrm{y}$ will not be compared to $\mathrm{x}$ anymore.

To avoid over fitting for the parameter search a 2 -fold validation was employed. Each fold contained the images from half of the subjects, and the subjects assigned to each fold were picked randomly. For each parameter set the EER was calculated for each fold. The comparison scores of a parameter set in fold 2 were selected based on the lowest EER value found in fold 1 and vice versa. Those selected comparison scores were combined and the EER was

\footnotetext{
${ }^{5}$ https://www.innovatrics.com/awards/m-o-c/

${ }^{6} \mathrm{https}: / /$ www.neurotechnology.com/megamatcher-on-card.html

${ }^{7}$ http://bias.csr.unibo.it/fvc2006/perfeval.asp
}

evaluated on them. The reason to do so is the following: After splitting the data into 2 folds, the amount of comparisons is reduced, especially the genuine comparisons. In case of the UTFVP data set for example in each fold only 1080 genuine and 16110 impostor comparison scores are computed. Therefore, the selected scores of both folds are combined to double the amount of comparison results for the final EER computation. The assumption for combining the scores is, that their distributions exhibit similar behavior across different settings and folds. To report the results with a statistical relevance, the calculation of the performance values using the procedure described above has been applied $N=100$ times, each time the subjects assigned to a fold were randomly picked. The same $N$ 2-fold splits for a data set have been used across all recognition methods.

To identify a best setting for each method and database those settings which produced the best EER in each fold have been counted. The setting with the highest count over all $N$ fold splits was considered to be the best parameter set. With these best settings a full comparison evaluation has been conducted, meaning within a database each sample was compared to all other samples. In case of the UTFVP database 2160 genuine and 1033920 impostor comparison scores would be computed. This has been done to increase the number of comparisons and to confirm the method's performance results.

Evaluation of time consumption: Template comparison execution times have been evaluated to reveal performance differences between the minutiae-based and classical vein recognition techniques. A set of templates was loaded into the RAM and then all comparisons were executed subsequently. VeriFinger and IDKit provide CSharp dotNet libraries. The PC, GF, ASAVE, MHD and SML method were implemented in $\mathrm{C}++(\mathrm{Cxx} 11)$ using the OpenCV library 3.4.2 and the Microsoft (R) $\mathrm{C} / \mathrm{C}++$ Optimizing Compiler Version 19.15.26732.1. The code has been compiled on optimization level $\backslash \mathrm{O} 2$. The tests have been executed on a 64-Bit personal computer with an Intel(R) Core(TM) i5-2400 CPU @ 3.10 GHz and Windows 10 Pro Version 1903 as operating system.

Software and used settings are made publicly available ${ }^{8}$.

\section{RESULTS}

Table 1 - 4 report the recognition performance for the used data sets and methods applying the 2-fold evaluation described in the previous section. EER and area under ROC curve (AUC) are reported. The ROC curve is defined as follows: the false match rate (FMR) is plotted along the abscissa against the false non-match rate (FNMR) on the ordinate. For all values the average (avg) and standard deviation (std) have been calculated using the results of the $N=100$ different fold splits. The minimum and maximum EER values are presented to show how the performance can vary depending on the selection of subjects for the parameter estimation.

With the three minutiae-based comparison tools Bozorth3, IDKit and VeriFinger all minutiae sets have been evaluated twice: one time with minutiae direction information $\theta$ present and one time with $\theta$ set to zero for each minutiae point. Throughout all tables the methods are marked with * where the results have been produced with minutiae sets where $\theta=0^{\circ}$ for all minutiae points. The other

\footnotetext{
${ }^{8}$ http://wavelab.at/sources/Linortner21a/
} 
Table 1: Recognition performance (EER and AUC) for the UTFVP data set using 2 -fold evaluation. Methods marked with * indicate that minutiae orientation $\theta$ is set to zero.

\begin{tabular}{lrrrrrr} 
UTFVP & \multicolumn{3}{c}{ EER in\% } & \multicolumn{2}{c}{ AUC in\% } \\
& avg & std & min & max & avg & std \\
\hline Bozorth3 $^{2.4}$ & 0.4 & 2.0 & 4.1 & 0.47 & 0.09 \\
Bozorth3 $^{*}$ & 13.4 & 0.6 & 12.1 & 15.6 & 7.52 & 0.56 \\
IDKit $^{\text {IDKit }}$ & 2.2 & 0.3 & 1.7 & 3.2 & 0.48 & 0.11 \\
VeriFinger $^{*}$ & 1.2 & 0.2 & 1.0 & 2.1 & 0.13 & 0.04 \\
VeriFinger & 3.0 & 0.2 & 2.6 & 3.6 & 0.46 & 0.06 \\
MHD & 0.5 & 0.1 & 0.3 & 0.6 & 0.02 & 0.01 \\
SML & 11.4 & 0.5 & 11.0 & 13.8 & 4.81 & 0.36 \\
PC & 4.7 & 1.0 & 3.7 & 7.4 & 1.19 & 0.37 \\
GF & 0.5 & 0.2 & 0.2 & 1.3 & 0.15 & 0.06 \\
ASAVE & 1.7 & 0.2 & 1.6 & 2.5 & 0.40 & 0.03 \\
& 2.0 & 0.4 & 1.4 & 2.8 & 0.40 & 0.14
\end{tabular}

Table 2: Recognition performance (EER and AUC) for the PLUS-Led-P data set using 2-fold evaluation.

\begin{tabular}{lrrrrrr} 
PLUS-Led-P & \multicolumn{3}{c}{ EER in\% } & \multicolumn{2}{c}{ AUC in\% } \\
& avg & std & min & max & avg & std \\
\hline Bozorth3 1.7 & 0.3 & 1.3 & 2.7 & 0.32 & 0.09 \\
Bozorth3* $^{*}$ & 12.2 & 0.4 & 11.4 & 13.7 & 5.67 & 0.32 \\
IDKit $^{\text {IDKit* }}$ & 1.4 & 0.4 & 1.1 & 3.4 & 0.38 & 0.10 \\
VeriFinger $^{*}$ & 2.2 & 0.6 & 1.7 & 5.0 & 0.48 & 0.16 \\
VeriFinger $^{*}$ & 1.2 & 0.1 & 0.9 & 1.6 & 0.11 & 0.02 \\
MHD & 0.3 & 0.1 & 0.2 & 0.6 & 0.02 & 0.01 \\
SML & 12.5 & 0.6 & 11.8 & 14.9 & 5.51 & 0.38 \\
PC & 2.6 & 0.3 & 2.2 & 3.5 & 0.47 & 0.12 \\
GF & 1.2 & 0.2 & 0.8 & 2.4 & 0.30 & 0.10 \\
ASAVE & 2.4 & 0.5 & 1.9 & 4.2 & 0.53 & 0.26 \\
& 1.5 & 0.2 & 1.3 & 2.1 & 0.30 & 0.04
\end{tabular}

Table 3: Recognition performance (EER and AUC) for the PLUS-Led-D data set using 2-fold evaluation.

\begin{tabular}{lrrrrrr} 
PLUS-Led-D & \multicolumn{4}{c}{ EER in\% } & \multicolumn{2}{c}{ AUC in\% } \\
& avg & std & min & max & avg & std \\
\hline Bozorth3 $^{*} 0.9$ & 0.2 & 0.6 & 1.4 & 0.15 & 0.03 \\
Bozorth3 $^{*}$ & 9.8 & 0.3 & 9.1 & 11.0 & 4.07 & 0.19 \\
IDKit & 1.2 & 0.3 & 0.8 & 2.5 & 0.18 & 0.08 \\
IDKit $^{*}$ & 1.4 & 0.2 & 1.0 & 2.1 & 0.17 & 0.05 \\
VeriFinger $^{*}$ & 0.7 & 0.1 & 0.5 & 1.0 & 0.05 & 0.02 \\
VeriFinger & 0.1 & 0.0 & 0.0 & 0.2 & 0.00 & 0.00 \\
MHD & 9.2 & 1.8 & 7.1 & 18.2 & 3.33 & 1.00 \\
SML & 1.6 & 0.6 & 1.1 & 5.6 & 0.24 & 0.20 \\
PC & 0.5 & 0.2 & 0.3 & 1.0 & 0.06 & 0.03 \\
GF & 1.2 & 0.3 & 0.9 & 2.4 & 0.17 & 0.11 \\
ASAVE & 0.4 & 0.1 & 0.2 & 0.9 & 0.02 & 0.01
\end{tabular}

Table 4: Recognition performance (EER and AUC) for the HKPU-FV data set using 2-fold evaluation.

\begin{tabular}{lrrrrrr} 
HKPU-FV & \multicolumn{3}{c}{ EER in\% } & \multicolumn{2}{c}{ AUC in\% } \\
& avg & std & min & max & avg & std \\
\hline Bozorth3 & 11.5 & 0.5 & 10.8 & 12.9 & 5.47 & 0.35 \\
Bozorth3* $^{*}$ & 13.9 & 1.2 & 12.6 & 17.8 & 7.17 & 0.75 \\
IDKit $^{*}$ & 10.4 & 0.3 & 9.7 & 11.9 & 4.49 & 0.22 \\
IDKit $^{*}$ & 8.5 & 1.3 & 6.9 & 12.7 & 3.39 & 0.67 \\
VeriFinger $^{*}$ & 10.6 & 0.3 & 10.1 & 11.8 & 3.77 & 0.17 \\
VeriFinger $^{*}$ & 2.8 & 0.2 & 2.5 & 3.7 & 0.50 & 0.04 \\
MHD & 11.6 & 1.3 & 9.9 & 16.2 & 5.32 & 0.82 \\
SML & 8.5 & 0.4 & 7.7 & 9.4 & 2.96 & 0.27 \\
PC & 2.0 & 0.3 & 1.6 & 3.1 & 0.51 & 0.11 \\
GF & 2.9 & 0.2 & 2.8 & 3.4 & 0.76 & 0.05 \\
ASAVE & 3.3 & 0.2 & 2.9 & 3.8 & 0.85 & 0.09
\end{tabular}

two minutiae-based methods MHD and SML solely use location information per design and hence they were evaluated only once. The three fingerprint comparison tools showed a quite contrary behavior regarding the minutiae orientation $\theta$ being available or not.
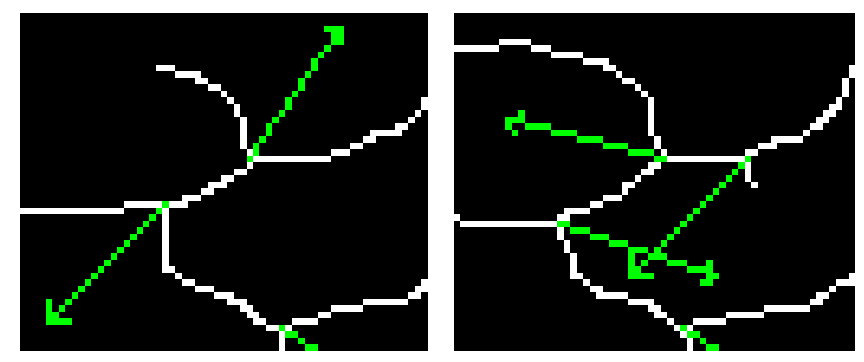

Figure 3: The two image details above show the same extracted vein structure in two different genuine samples. The orientation, indicated by the arrows, of the two top left minutiae bifurcation points flips due to small variations in the enclosed angles between the branches. This may cause a performance drop if it happens at too many minutia points.

On all data sets VeriFinger achieved better results in terms of EER using minutiae sets with $\theta=0^{\circ}$, in three cases the performance increased about a magnitude. The IDKit showed a notable performance increase with $\theta=0^{\circ}$ on the UTFVP and HKPU-FV database but a decline on the PLUS-Led databases. Bozorth3 on the other hand showed a clear performance drop when no minutiae orientation was available. Even the more naive MHD approach generated slightly better results then. This indicates, for Bozorth3 the minutiae orientation is crucial. For the other two tools it was somehow unexpected that dropping the orientation information increased the performance, since it is a defining element of a minutiae point. A possible explanation may be given, looking at the appearance of a bifurcation in the vein structure compared to those in a fingerprint. The gap of two branches of a ridge at a fingerprint bifurcation point usually is narrow and so the difference between the smallest enclosed angle and the two other angles is clearly 
Table 5: Recognition performance for all data set using full comparison protocol and best settings. All values are in \%.

\begin{tabular}{llrrrrrr} 
Data set & Method & EER & FMR100 & ZeroFMR & FNMR100 & ZeroFNMR & AUC \\
\hline UTFVP & VeriFinger $^{*}$ & 0.28 & 0.20 & 3.29 & 0.02 & 13.99 & 0.01 \\
& PC & 0.23 & 0.19 & 0.56 & 0.00 & 64.43 & 0.06 \\
PLUS-Led-P & VeriFinger $^{*}$ & 0.17 & 0.16 & 1.22 & 0.00 & 14.24 & 0.01 \\
& PC & 0.82 & 0.78 & 3.92 & 0.48 & 82.16 & 0.19 \\
\multirow{2}{*}{ PLUS-Led-D } & VeriFinger $^{*}$ & 0.04 & 0.03 & 0.39 & 0.00 & 2.23 & 0.00 \\
& PC & 0.28 & 0.22 & 1.39 & 0.00 & 42.32 & 0.05 \\
HKPU-FV & VeriFinger $^{*}$ & 2.41 & 3.27 & 24.98 & 12.65 & 48.87 & 0.41 \\
& PC & 1.41 & 1.58 & 9.21 & 4.06 & 99.53 & 0.32
\end{tabular}

recognizable. In the case of vein minutiae the angles between vein branches at a bifurcation point are often wider. It can happen that at the same minutiae point in two different genuine samples the identified smallest enclosed angle appears between different vein branches. As a consequence the orientation of the minutiae points flips between genuine samples. Figure 3 illustrates this issue with an example vein structure detail obtained from two genuine samples of the UTFVP database. If the flipping happens on too many minutia points the available orientation information may cause the performance drop compared to using the minutiae point location with a fixed orientation value.

Bozorth3 performed at a comparable level with IDKit and on the PLUS-Led-D data set it achieved even better results. It was not expected that the publicly available Bozorth3 is able to compete with a commercial product in this scenario, since its performance is worse in comparison on fingerprint data [9]. A possible explanation is, that VeriFinger and IDKit usually produce their own (proprietary) templates with additional information from input fingerprint images. In this scenario the same minutiae with only the location and direction information are given as input to the comparison modules.

The other both minutia-based methods SML and MHD showed a lower performance and MHD, being a more naive approach, clearly resulted in significantly higher error rates for all databases. On the PLUS-Led-D data set SML performed better and came close to the results achieved by IDKit.

The three classical correlation-based vein recognition techniques used as reference comparison showed on all databases good performance. PC performed best, only at the PLUS-Led-D data set ASAVE achieved slightly better results.

In [19] a Squeeze-Net with triplet loss, a CNN approach, yielded an EER of $2.5 \%$ for UTFVP and $2.4 \%$ for PLUS-Led-P data set, which is a lower performance than Bozorth3, IDKit and VeriFinger achieved. On the HKPU-FV database it produced an EER of 3.7\%, which is lower than IDKit and Bozorth achieved here, but higher than VeriFingers $2.8 \%$ with $\theta=0^{\circ}$. The PLUS-Led-D data set has not been used in [19].

On the HKPU database the accuracy of the minutiae-based approach dropped significantly (only VeriFinger with $\theta=0^{\circ}$ achieved better results). One reason could be that this database contains a higher number of subjects (more than double compared to the other both databases). On the other hand, in these experiments each finger is considered as a single class, which results in 312 classes for HKPU compared to the 360 from UTFVP and PLUS databases.
It is more likely that the performance drop is caused by the image quality of the HKPU samples. In several images parts of the fingers are overexposed and no vein structure is visible at all. This non-ideal acquisition set-up my be caused by the absence of some guiding-structure to help the user to put the finger into an optimal position for the image acquisition. Results indicate that this is a major problem for the minutiae-based approach, whereas PC, GF and ASAVE can deal better with this situation. If too many minutiae points are lost due to low image quality it is difficult to achieve high accuracy.

From the reported minimal and maximal values of the EER it can be observed that the EER varies noticeably in most of the cases, depending on the subjects present in a data set or, as here, how the subjects were split into the folds.

Compared to the results in [5] our proposed feature extraction approach achieved a considerable improvement on the EER using the same evaluation protocol. This performance increase may be caused by either that the approach proposed here extracts more reliable and stable minutiae points or by just an increased number of minutiae points found.

Additional to the 2-fold a full comparison evaluation on the best settings has been conducted on all data sets. The full comparison protocol has been applied to confirm the results of the 2-fold evaluation based on an higher amount of comparison scores. For details see section 3. The results are listed in table 5. Only the best of the minutiae-based comparison and the classical correlation-based methods are listed. Next to EER and AUC the ZeroFMR, ZeroFNMR, FMR100 and FNMR100 were computed. ZeroFMR describes the lowest FNMR at a FMR of zero. FMR100 is the lowest FNMR for FMR $\leq 1 \%$. ZeroFNMR then is the lowest FMR at FNMR of zero and FNMR100 the lowest FMR for FNMR $\leq 1 \%$. All methods showed a slightly better performance than in the 2 -fold evaluation. It might be the score combining strategy for the 2 -fold evaluation is the reason for higher error rates. The results in table 5 show that on the PLUS data sets VeriFinger performed best. On the UTFVP database the minutiae-based method and the classical technique performed almost equally. On the HKPU-FV data set PC showed better results than VeriFinger, which indicates the minutia-based approach is more sensitive to the quality of the presented biometric samples.

Table 6 shows the average execution times for 66780 (2160 genuine and 64620 impostor) template comparisons using the UTFVP data set and using the best setting for each method. The experiments have been carried out 10 times and the average time is presented. The Bozorth3 algorithm is not considered here, because 
Table 6: Execution times of 66780 template comparisons.

\begin{tabular}{llr} 
& Method & Time in sec \\
\hline VeriFinger $^{*}$ & (minutiae-based) & $70.1 \pm 0.5$ \\
IDKit* $^{*}$ & (minutiae-based) & $369.4 \pm 1.7$ \\
MHD & (minutiae-based) & $61.1 \pm 0.6$ \\
SML & (min.-based / corr. + shifts) & $52.5 \pm 0.5$ \\
PC & (correlation + shifts) & $340.9 \pm 3.6$ \\
GF & (correlation + shifts) & $4044.6 \pm 94.6$ \\
ASAVE & (correlation + shifts) & $562.8 \pm 22.6$
\end{tabular}

in its publicly available implementation the time for comparing and reading a template from disk cannot be separated. PC, GF and ASAVE used a template size of $336 \times 128$ data points (size of the ROI image). The shifts applied on the templates to compensate for misalignment are $10 \%$ of the template width for horizontal and $15 \%$ of the height for vertical shifts. The results clearly show that the minutia-based methods work about one order of magnitude faster than the classical vein recognition methods. Only IDKit is slower and its performance falls into the same range as PC and ASAVE. Although SML uses correlation to obtain a similarity score, the template size is smaller and only a small shift in one direction needed to be applied, and therefore it is faster. ASAVE needs to correlate two binary templates internally to find a similarity score which results in a lower performance than PC. A main reason for GF being that much slower than PC and ASAVE is, that for the latter methods correlation and shifting can be combined exploiting some optimized functionality of the OpenCV library, whereas for GF this wasn't possible. In terms of complexity and computational effort those comparison methods should be equal otherwise.

\section{CONCLUSION}

In this work the feasibility of vein recognition towards Match-onCard (MoC) technology is shown: Minutiae points are extracted from vein images and stored in a standardized format which is needed to use classical minutiae-based fingerprint comparison tools for the recognition task. This is a necessary first step for a seamless integration of vein recognition for $\mathrm{MoC}$ systems. Two state of the art commercial and a publicly available minutiae-based fingerprint comparison software tools have been used. The results show that the minutiae-based techniques can not only compete with but also can outperform classical correlation-based and CNN-based approaches in terms of recognition performance. Considering comparison execution time the minutiae-based methods clearly have an additional advantage. The results have been considerably improved compared to those in a previous work [5].

The outcome of this work definitively motivates further research in minutiae-based techniques for vein recognition, especially towards the application of vein recognition in a MoC scenario.

\section{ACKNOWLEDGMENTS}

This project was partly funded by the Austrian Science Fund FWF project "Advanced Methods and Applications for Fingervein Recognition” under grant No. P 32201-NBL.

\section{REFERENCES}

[1] ANSI-INCITS 378-2004. 2004. Information Technology - Finger Minutiae Format for Data Interchange

[2] C. Arcelli and G. S. Di Baja. 1985. A Width-Independent Fast Thinning Algorithm. IEEE Trans. on Pattern Analysis and Machine Intelligence PAMI-7, 4 (July 1985), 463-474.

[3] A. M. R. R. Bandara, K. A. S. H. K. Rajarata, and P. W. G. R. M. P. B. Giragama. 2017. Super-efficient spatially adaptive contrast enhancement algorithm for superficial vein imaging. In 2017 IEEE Int. Conf. on Industrial and Inf. Systems (ICIIS). 1-6.

[4] Christer Bergman. 2008. Match-on-Card for Secure and Scalable Biometric Authentication. Springer London, London, 407-421.

[5] Katy Castillo-Rosado, Michael Linortner, Andreas Uhl, Heydi Mendez-Vasquez, and José Hernandez-Palancar. 2020. Minutiae-based Finger Vein Recognition Evaluated with Fingerprint Comparison Software. In Proceedings of the IEEE 19th Int. Conf. of the Biometrics Special Interest Group (BIOSIG 2020). Darmstadt, Germany, 1-8.

[6] Tai Pang Chen, Wei-Yun Yau, and Xudong Jiang. 2013. ISO/IEC standards for on-card biometric comparison. Int. fournal of Biometrics 5, 1 (2013), 30-52.

[7] Joon Hwan Choi, Wonseok Song, Taejeong Kim, Seung-Rae Lee, and Hee Chan Kim. 2009. Finger vein extraction using gradient normalization and principal curvature. In Image Processing: Machine Vision Applications II, Kurt S. Niel and David Fofi (Eds.), Vol. 7251. Int. Society for Optics and Photonics, SPIE, 359 367

[8] Christof Kauba, Bernhard Prommegger, and Andreas Uhl. 2018. The Two Sides of the Finger - An Evaluation on the Recognition Performance of Dorsal vs. Palmar Finger-Veins. In Proceedings of the Int. Conf. of the Biometrics Special Interest Group (BIOSIG'18). Darmstadt, Germany, 1-8.

[9] Simon Kirchgasser, Katy Castillo-Rosado, David Estevez-Breso, Emilio RodriguezHernandez, Jose Hernandez-Palancar, and Andreas Uhl. 2018. Fingerprint Template Ageing Revisited - It's the Quality, Stupid !. In Proceedings of the IEEE 9th Int. Conf. on Biometrics: Theory, Applications, and Systems (BTAS2018). Los Angeles, California, USA, 1-8.

[10] A. Kumar and Y. Zhou. 2012. Human Identification Using Finger Images. IEEE Trans. on Image Processing 21, 4 (April 2012), 2228-2244.

[11] Fei Liu, Gongping Yang, Yilong Yin, and Shuaiqiang Wang. 2014. Singular value decomposition based minutiae matching method for finger vein recognition. Neurocomputing 145 (2014), 75 - 89.

[12] Yu Lu, Shan Juan Xie, Sook Yoon, Jucheng Yang, and Dong Sun Park. 2013. Robust finger vein ROI localization based on flexible segmentation. Sensors 13, 11 (2013), 14339-14366.

[13] Davide Maltoni, Dario Maio, Anil K Jain, and Salil Prabhakar. 2009. Handbook of fingerprint recognition. Springer Science \& Business Media.

[14] T. S. Ong, J. H. Teng, K. S. Muthu, and A. B. J. Teoh. 2013. Multi-instance finger vein recognition using minutiae matching. In 2013 th Int. Congress on Image and Signal Processing (CISP), Vol. 03. 1730-1735.

[15] R. Raghavendra, K. B. Raja, J. Surbiryala, and C. Busch. 2014. A low-cost multimodal biometric sensor to capture finger vein and fingerprint. In IEEE Int. Foint Conf. on Biometrics. 1-7.

[16] Sung Bum Pan, Daesung Moon, Younhee Gil, Dosung Ahn, and Yongwha Chung. 2003. An ultra-low memory fingerprint matching algorithm and its implementation on a 32-bit smart card. IEEE Trans. on Consumer Electronics 49, 2 (2003), 453-459.

[17] B. T. Ton and R. N. J. Veldhuis. 2013. A high quality finger vascular pattern dataset collected using a custom designed capturing device. In 2013 Int. Conf. on Biometrics (ICB). 1-5.

[18] Andreas Uhl, Christoph Busch, Sebastien Marcel, and Raymond Veldhuis. 2020. Handbook of Vascular Biometrics. Springer Nature Switzerland AG, Cham, Switzerland.

[19] Georg Wimmer, Bernhard Prommegger, and Andreas Uhl. 2020. Finger Vein Recognition and Intra-Subject Similarity Evaluation of Finger Veins using the CNN Triplet Loss. In Proceedings of the 25th Int. Conf. on Pattern Recognition (ICPR). 1-7. accepted.

[20] Haiyun Xu, R. N. J. Veldhuis, T. A. M. Kevenaar, A. H. M. Akkermans, and A. M. Bazen. 2008. Spectral minutiae: A fixed-length representation of a minutiae set. In 2008 IEEE Computer Society Conf. on Computer Vision and Pattern Recognition Workshops. 1-6.

[21] L. Yang, G. Yang, X. Xi, X. Meng, C. Zhang, and Y. Yin. 2017. Tri-Branch Vein Structure Assisted Finger Vein Recognition. IEEE Access 5 (2017), 21020-21028.

[22] L. Yang, G. Yang, Y. Yin, and X. Xi. 2017. Finger Vein Recognition with Anatomy Structure Analysis. IEEE Trans. on Circuits and Systems for Video Technology (2017), 1-1.

[23] Cheng-Bo Yu, Hua-Feng Qin, Yan-Zhe Cui, and Xiao-Qian Hu. 2009. Finger-vein image recognition combining modified Hausdorff distance with minutiae feature matching. Interdisciplinary Sciences: Computational Life Sciences 1, 4 (Dec 2009), 280-289.

[24] T. Y. Zhang and C. Y. Suen. 1984. A Fast Parallel Algorithm for Thinning Digital Patterns. Commun. ACM 27, 3 (March 1984), 236-239. 